\title{
Asociación de los factores motivacionales y el desempeño laboral del tecnólogo médico en radiología *
}

\author{
Association Between Motivating Factors and Employee Performance of \\ Radiology Technicians
}

\author{
Guillermo Alex Guzmán-Narciso (iD) \\ Tecnólogo Médico, Universidad Nacional Mayor de San Marcos, \\ Lima-Perú,gagn990566@hotmail.com \\ Waynner Sánchez-García \\ Tecnólogo Médico, Universidad Nacional Mayor de San Marcos, \\ Lima-Perú, waynnersg@gmail.com \\ Maritza Dorila Placencia-Medina \\ Doctora en Farmacia y Bioquímica, Universidad Nacional Mayor de San Marcos, \\ Lima-Perú, mplacenciam@unmsm.edu.pe \\ Javier Silva-Valencia \\ Médico Cirujano e Informático Biomédico, Universidad Nacional Mayor de San Marcos, \\ Lima-Perú, javsil256@gmail.com \\ Guzmán-Narciso, G. A.; Sánchez-García, W.; Placencia-Medina, M. D.; Silva-Valencia, J. (2020). Asociación de \\ los factores motivacionales y el desempeño laboral del tecnólogo médico en radiología. Revista CEA, v. 6, \\ n. 12, 87-103. https://doi.org/10.22430/24223182.1543
}

Cómo citar / How to cite

Recibido: 4 de febrero de 2020

Aceptado: 6 de mayo de 2020

\section{Resumen}

El objetivo de este trabajo fue determinar la asociación entre los factores motivacionales y el desempeño laboral, en los tecnólogos médicos en el área de radiología. Para esto se realizó un estudio descriptivo de corte transversal, analítico correlacional, en el cual se utilizaron como instrumentos de recolección de datos el «Test de evaluación de factores motivacionales» (para medir

\footnotetext{
* Este artículo se deriva del proyecto del proyecto de tesis en Maestría en Gerencia de Servicios de Salud, titulado «Factores motivacionales asociados al desempeño laboral del tecnólogo médico en el área de radiología, seguro social de salud, Essalud- 2016» y ha sido financiado con recursos propios.
} 
los factores motivacionales según F. Herzberg) y la «Ficha de Evaluación del desempeño laboral» (para medir el desempeño laboral de los participantes, según evaluación de su jefatura). Posteriormente, se determinó la asociación entre los FM y el desempeño laboral. Para el análisis estadístico se utilizó el programa Microsoft Excel, programa estadístico Stata v.16.0 y la correlación de Pearson. La población y la muestra estuvo compuesta por $\mathrm{N}=77$ y $\mathrm{n}=64$ participantes, respectivamente. Entre los factores motivacionales más resaltantes fueron el crecimiento profesional, relaciones con los compañeros, logro y seguridad laboral, con puntajes promedio de $9.015 \pm 1.923,8.812 \pm 2.259,8.406 \pm 2.060$ y $8.203 \pm 2.520$, respectivamente. A su vez, el desempeño laboral obtenido fue regular (7.81\%), bueno (28.13\%) y muy bueno (64.06\%). Con respecto a la asociación existente entre factores motivacionales y desempeño laboral, de los 15 factores motivacionales estudiados, los valores de mayor relevancia fueron obtenidos por «seguridad laboral» ( $r$ : 0.2511, $p=0.04)$ y «relaciones con el jefe» $(r:-0.2508, p=0.04)$. Se concluye que Los factores motivacionales «seguridad laboral» y «relaciones con el jefe» tienen una asociación significativa con el desempeño laboral en los TM del área de radiología. Donde se evidenció que, a mayor priorización de los factores motivacionales de las relaciones con el jefe, menor es el nivel de desempeño laboral; y que contrariamente, a mayor priorización de los factores motivacionales, mayor es el nivel de desempeño laboral.

Palabras clave: factores motivacionales; desempeño laboral; talento humano, personal médico.

Clasificación JEL: I19; M54.

\section{Abstract}

The objective of this study was to determine the association between the Motivating Factors (MFs) and employee performance of radiology technicians. The methodology was a cross-sectional, descriptive correlation analysis that used a "Test to evaluate Motivating Factors" (according to F. Herzberg) and a "Performance review sheet" (to measure the performance of the participants, according to their supervisors) to collect data. Afterward, we determined the association between MFs and employee performance. We used Microsoft Excel, Stata v.16.0, and the Pearson correlation for the statistical analysis. The population and the sample comprised $\mathrm{N}=77$ and $\mathrm{n}=64$ participants, respectively. Among the most remarkable motivating factors, we found that the average scores of professional growth, relationships with peers, achievement, and work security were $9.015 \pm 1.923$, $8.812 \pm 2.259,8.406 \pm 2.060$, and $8.203 \pm 2.520$, respectively. In turn, employee performance was unsatisfactory $(7.81 \%)$, good $(28.13 \%)$, and very good (64.06\%). Regarding the association between motivating factors and employee performance, out of the 15 motivating factors studied here, the most relevant values were those of "work security" ( $r$ : 0.2511, p=0.04) and "relationship with supervision" ( $r$ : $-0.2508, p=0.04)$. We conclude that these two motivating factors are significantly associated with the employee performance of radiology technicians. We also found that, the higher the priority of the relationship with supervisors, the worse the employee performance; and, on the contrary, the higher the priority of motivating factors, the better the performance.

Keywords: motivating factors, employee performance, human talent, health professionals.

JEL classification: I19; M54. 


\section{INTRODUCCIÓN}

La búsqueda para mejorar el desempeño laboral (DL) dentro de las organizaciones es una prioridad necesaria para encaminar el éxito de las mismas (Lusthaus et al., 2002; Silva Rodríguez de San Miguel, 2018; Jiang et al., 2019). Palaci Descals (2005) plantea que el desempeño laboral es el valor esperado como aporte a la organización en un periodo de tiempo. Estas conductas, de un mismo o varios individuos, en diferentes momentos temporales a la vez, contribuirán a la eficiencia organizacional, y el desarrollo de estos puede ser motivado por diferentes factores personales o ambientales, que buscan el perfeccionamiento de su liderazgo y sus habilidades (Arana Mayorca, 2004; Uribe-Gómez, 2018; Billyard \& Donohue, 2016).

La motivación es un proceso que da cuenta de la intensidad, dirección y persistencia del esfuerzo por conseguir una meta (Robbins, 2004; Alvarado Arévalo, 2017), que al parecer está bajo el control de las personas (Vázquez-Moctezuma, 2014) y que requiere de otros enfoques que tomen en cuenta tanto factores personales como ambientales (López Más, 2005; Yunus et al., 2020).

Uno de esos enfoques es la teoría bifactorial de Frederick Herzberg, la cual evidencia que los FM se dividen en extrínsecos (factores de higiene) e intrínsecos (factores motivacionales) (Peiró \& Meliá, 2003). Los FM extrínsecos reflejan un carácter preventivo y profiláctico, si son excelentes solo evitan la insatisfacción, pues su influencia en el comportamiento no consigue elevar en forma sustancial ni duradera la satisfacción de las personas; sin embargo, cuando son precarios provocan insatisfacción; están relacionados al ambiente que rodea a las personas y abarcan las condiciones en que desempeñan su trabajo (Martin \& Sorhaindo, 2019). Los FM extrínsecos son condiciones de trabajo, políticas administrativas de la empresa, sueldo, las relaciones con el jefe, relaciones con sus compañeros de trabajo, supervisión, vida personal, prestigio o estatus y seguridad de trabajo (Chiavenato, 2009). Por otro lado, los FM intrínsecos están relacionados directamente a la satisfacción del trabajador en sí mismo, priorizando la capacidad de respuesta frente al reto emprendido, aún más que la remuneración salarial recibida; dentro de estos factores se tiene el reconocimiento profesional, trabajo en sí mismo, responsabilidad, crecimiento profesional, asenso o promoción y logro. A mayor grado de motivación laboral, mayor grado de satisfacción laboral del personal. La relación entre la motivación y satisfacción laboral es de correlación positiva, aunque de baja intensidad. (Marín Samanez \& Placencia Medina, 2017; Franco-López \& Bedoya-Zapata, 2018).

La relación entre los FM y el DL es directamente proporcional (Linares Marín, 2017) y se ha visto que depende de las metas, la satisfacción de necesidades y una mente positiva hacia la actividad laboral, que se convierten en la fortaleza que activa la motivación, que proporciona la disposición necesaria para un mejor desarrollo de las actividades laborales (García Santillán \& Uscanga Guevara, 2008).

Específicamente, en el área de la salud, por trabajar con seres humanos, el entorno laboral está sujeto a condiciones ambientales, estrés, presión (Organización Mundial de la Salud, 2010; Vänje, 2015) y se ha visto que los FM pueden ser muy variados, predominando principalmente: condiciones de trabajo, salario, relaciones con los compañeros, supervisión, seguridad, logro, reconocimiento y responsabilidad (Arana Mayorca, 2004). Existen estudios que relacionan un deficiente desempeño laboral con bajos niveles de FM intrínsecos y extrínsecos (Campos Huarez, 2018) y otro que menciona altos niveles de FM intrínsecos frente al DL predominante, obteniendo una moderada relación (Bobadilla Asenjo, 2016). 
Existen múltiples trabajos de investigación en instituciones de salud cuyos resultados en profesionales de salud están asociados a los FM y el DL (Arias Gonzales, 2019; Salcedo Ramos, 2020; Ríos Valdera, 2017; Román Meza, 2016; Paredes Cabanillas, 2017); sin embargo, estos resultados no se pueden generalizar para todos los profesionales de salud.

Las características asistenciales del TM en radiología son distintas a las de otros profesionales y propias de su área, debido a que se desenvuelve en un entorno de constante evolución tecnológica (equipos y programas radiológicos nuevos), exposición a radiaciones ionizantes, manipulación de imágenes y manejo de medicamentos de alto riesgo (Anderson et al., 2016); es en este contexto donde la motivación toma gran importancia, ya que para para alcanzar los objetivos de las organizaciones es necesario que sus líderes tengan bien claro cuáles son los FM que contribuyen, en mayor o menor grado, a un excelente DL, que a la larga contribuirá a una mayor productividad. Sus características tecnológicas de vanguardia hacen que experimente diferentes FM durante el desarrollo de sus actividades laborales (Nojedeh, 2015).

En este contexto el objetivo del estudio fue determinar la asociación existente entre los FM del profesional TM con el DL, aplicando un cuestionario de FM basados en la teoría bifactorial de Frederick Herzberg y la ficha de evaluación del DL calificado por el jefe inmediato superior (ficha institucional que caracteriza el DL del TM en el área de radiología). Muchas jefaturas suelen creer que el contenido del trabajo es constante; sin embargo, los trabajos sí se pueden modificar y enriquecer. Los jefes deberían enfocarse en los puestos donde las actitudes de los trabajadores no contribuyen positivamente para alcanzar los objetivos de la organización, la inversión necesaria en cualquier organización es efectiva en cuanto a costos, pero además si se invierte en motivación habrá una diferencia positiva en el desempeño laboral de los empleados, que hará más fácil la obtención de resultados esperados (Herzberg, 2003).

Los resultados de este estudio servirán como insumo de mejora continua a las autoridades de Gestión de Recursos Humanos, para establecer estrategias motivacionales y para contribuir en la mejora del $\mathrm{DL}$, en el ámbito laboral y social.

\section{METOdOLOGÍA}

Estudio cuantitativo, descriptivo de corte transversal y correlacional que estudió los FM y el nivel de DL en la población de Tecnólogos Médicos (TM) en el área de radiología de dos hospitales de alta complejidad en la capital de Perú. El escenario elegido para este trabajo fue el Seguro Social de Salud (EsSalud) en el Perú, el cual atiende a más de 10 millones de usuarios, entre asegurados y derechohabientes (EsSalud, 2016); específicamente se eligió el Hospital Edgardo Rebagliati Martins (HNERM) y el Hospital Guillermo Almenara Irigoyen (HNGAI), los cuales son los establecimientos de salud de más alta complejidad a nivel nacional.

Dichos hospitales son centros de referencia a nivel nacional y con gran demanda de atención de pacientes; teniendo por finalidad dar cobertura a los asegurados y sus derechohabientes, a través de la prestación servicios de prevención, promoción, recuperación, rehabilitación, prestaciones económicas y prestaciones sociales, así como otros seguros de riesgos humanos (EsSalud, 2020). 


\section{Población y muestra}

La población del estudio estuvo conformada por todos los profesionales TM en el área de radiología que laboraron durante el año 2016 en los hospitales HNERM y HNGAI (77 profesionales). Se realizó un cálculo de tamaño muestral utilizando una frecuencia anticipada de $50 \%$ y un nivel de confianza de $95 \%$. Mediante un muestreo aleatorio simple se invitó a participar a los TM y se incluyó aquellos que aceptaban participar voluntariamente y cumplían los criterios de selección hasta completar la muestra $(n=64)$

\section{Criterios de inclusión}

Profesional tecnólogo médico en el área de radiología (rayos $\mathrm{X}$, radiología intervencionista, tomografía computarizada y/o resonancia magnética), con edad menor o igual a 60 años, que labora en el HNERM o en el HNGAI y que aceptaron participar en la investigación.

\section{Criterios de exclusión}

Profesional tecnólogo médico cuyo tiempo de trabajo durante el año 2016 fue menor de dos meses, que labora en el HNERM o en el HNGAI.

\section{Cálculo del tamaño de muestra}

Muestra calculada con un índice de confiabilidad de $95 \%$ y un error de muestreo del $5 \%$ :

Ecuación para muestra finita:

$$
n=\frac{N Z^{2} p q}{d^{2}(N-1)+Z^{2} p q}
$$

Dónde:

Población (N): 77 trabajadores.

Confianza (Z): $95 \%=1.96$.

Prevalencia o probabilidad de éxito $(p): 50 \%=0.50$.

Prevalencia o probabilidad de fracaso $(q): 50 \%=0.50$.

Error permisible de muestreo (d): $5 \%=0.05$.

$$
n=\frac{77(1.96)^{2}(0.50)(0.50)}{0.05^{2}(77-1)+(1.96)^{2}(0.50)(0.50)}
$$

$n=64$

\section{Instrumentos para recolección de información:}

Se estudiaron dos variables principales: factores motivacionales como variable independiente y el desempeño laboral como variable dependiente. Para medir los factores motivacionales se aplicó el "Test de evaluación de factores motivacionales», el cual es un instrumento validado por Arana Mayorca en el año (2004), que cuenta con 105 preguntas para evaluar la preferencia de elección hacia 15 diferentes FM, divididos aleatoriamente en extrínsecos e intrínsecos. Cada pregunta considera 2 alternativas que representan el modo de razonar de cada persona. 
Para medir el desempeño laboral se usó la «Ficha de evaluación del DL»; esta ficha es el cuestionario oficial de la red de EsSalud en Lima-Metropolitana, aprobado por la Resolución de presidencia ejecutiva N 1046-PE-EsSalud-2004, y evalúa 8 criterios del DL, divididos en dos factores: factores de rendimiento como cumplimientos de metas asignadas y calidad de trabajo efectuado; $y$ factores de idoneidad para el puesto como compromiso y responsabilidad, habilidad para trabajar bajo presión, entre otros (EsSalud, 2004).

\section{Procedimiento}

Se inició el estudio validando la Ficha de desempeño laboral para su uso en esta investigación, por medio de un juicio de expertos en la dependencia de los hospitales en los cuales se realizó el estudio. Asimismo, luego de que el estudio fuera aprobado en los HNERM y HNGAl, se procedió al enrolamiento y recolección de datos.

Para la recolección de datos sobre los FM se abordó a los TM en el área de radiología de cada hospital en una reunión grupal, donde se les explicó el objetivo de la investigación y se les entregó el cuestionario el cual deberían completarlo para el día siguiente.

Para la recolección de datos sobre el DL se contactó con el jefe inmediato superior de cada profesional encuestado, el cual valoró al TM bajo codificación confidencial en su rendimiento e idoneidad para el puesto de trabajo desempeñado en el HNERM y el HNGAI. La recolección de datos se realizó por un periodo de tres meses.

\section{Consideraciones éticas}

Se entregó una hoja de consentimiento informado al empleado en la cual autorizaba su participación, dando expreso conocimiento de actuar libre y voluntariamente como colaborador. Se trabajó cumpliendo las normas éticas de investigación con seres humanos, entre ellas el principio de la autonomía de participación y la no maleficencia, la participación voluntaria y el no causar ningún riesgo a su integridad física y moral, así como el mantener en confidencialidad los datos que proporcionaron, evitando poner en riesgo su identificación y sobretodo codificando los datos en el uso exclusivo para el estudio.

\section{Análisis estadístico}

Se utilizó el programa Microsoft Excel para tabular y limpiar los datos. Asimismo, para el análisis descriptivo y analítico se usó el software estadístico Stata v.16.0. Las variables FM y DL son tratadas como variables categóricas para tener la valoración categórica en cada uno de ellos, y como variables numéricas para analizar la correlación entre el puntaje obtenido de ambas mediante el coeficiente de correlación de Pearson. Para todos los análisis se utilizó un nivel de significancia de 0.05.

\section{RESULTADOS}

Se obtuvo información de 64 TM. En la Tabla 1 se resumen los datos sociodemográficos. El 57.81\% fueron del género masculino y el 70.32\% estaba entre 31- 50 años. Las áreas donde se encontró más personal fue en las que tenían más contacto con los pacientes, como rayos $\mathrm{X}$ e intervencionismo, en 
comparación con áreas como resonancia magnética y tomografía donde la interacción es menor, pero el uso de tecnología digital computarizada es mayor.

Tabla 1. Identificación sociodemográfica del tecnólogo médico en el área de radiología del HNERM-HNGAI, 2016

Table 1. Sociodemographic profile of the radiology technicians at HNERM-HNGAI, 2016

\begin{tabular}{|c|c|}
\hline Características & $\%$ \\
\hline \multicolumn{2}{|l|}{ Género } \\
\hline Masculino & $57.81 \%$ \\
\hline Femenino & $42.19 \%$ \\
\hline \multicolumn{2}{|l|}{ Grupo etareo } \\
\hline$<30$ años & $4.68 \%$ \\
\hline 31-40 años & $34.38 \%$ \\
\hline 41-50 años & $35.94 \%$ \\
\hline$>50$ años & $25 \%$ \\
\hline \multicolumn{2}{|l|}{ Hospital donde labora } \\
\hline HNERM & $57.81 \%$ \\
\hline HNGAI & $42.19 \%$ \\
\hline \multicolumn{2}{|l|}{ Área específica } \\
\hline Rayos $\mathrm{x}$ - intervencionismo & $67.19 \%$ \\
\hline Resonancia Magnética-Tomografía & $32.81 \%$ \\
\hline \multicolumn{2}{|l|}{ Régimen laboral } \\
\hline Nombrado & $23.44 \%$ \\
\hline Contrato indeterminado & $57.81 \%$ \\
\hline $\begin{array}{l}\text { Contrato Administrativo de } \\
\text { Servicios }^{(1)}(\mathrm{CAS})\end{array}$ & $18.75 \%$ \\
\hline
\end{tabular}

Fuente: elaboración propia.

Cuando se evaluó los FM del comportamiento humano, se encontró que los TM consideraban más importantes los factores de (1) crecimiento profesional (potencial de ascenso progresivo profesional), (2) relaciones con los compañeros (relaciones interpersonales) y (3) logro (sensación de realización personal). Mientras que los FM menos importantes fueron estatus (sentimiento de prestigio dentro de la organización) y supervisión (relación entre el que supervisa y el que trabaja). Esta priorización fue independiente del hospital en donde se encontraban o del tipo de contrato que tenían (ver Tabla 2).

\footnotetext{
${ }^{1}$ CAS: es una modalidad contractual administrativa y privativa del estado peruano, que vincula a una entidad pública con una persona natural que presta servicios de manera no autónoma.
} 
Tabla 2. Puntaje de factores motivacionales del tecnólogo médico en el área de radiología, en HNERMHNGAI-2016, según la teoría Bifactorial de Frederick Herzberg

Table 2. Scores of motivating factors of radiology technicians at HNERM-HNGAI, 2016, according to Frederick Herzberg's two-factor theory

\begin{tabular}{lcc}
\multicolumn{1}{c}{ Factor motivacional } & Media & $\begin{array}{c}\text { Desviación } \\
\text { estándar }\end{array}$ \\
\hline Crecimiento profesional & 9.015 & 1.923 \\
Relaciones con los compañeros & 8.812 & 2.259 \\
Logro & 8.406 & 2.06 \\
Seguridad laboral & 8.203 & 2.52 \\
Condiciones de trabajo & 8.171 & 2.285 \\
Política y administración & 7.796 & 2.071 \\
Vida personal & 7.359 & 2.704 \\
Responsabilidad & 7.265 & 1.887 \\
Ascenso & 6.875 & 2.42 \\
El trabajo mismo & 6.765 & 1.883 \\
Sueldo & 6.64 & 2.796 \\
Reconocimiento & 6.562 & 1.78 \\
Relaciones con el jefe & 5.296 & 1.989 \\
Supervisión & 4.606 & 2.615 \\
Estatus & 3.666 & 2.03 \\
\hline
\end{tabular}

Fuente: elaboración propia.

Respecto al DL, el valor numérico obtenido se clasificó en 4 categorías según la ficha de evaluación del DL, siendo: malo, regular, bueno y muy bueno. No se encontró participante calificado con un desempeño laboral malo (0\%) y en general solo un $7.81 \%$ fue calificado de tener un DL regular, mientras que $28.13 \%$ fue calificado con DL bueno y $64.06 \%$ con DL muy bueno. En la Tabla 3 se puede ver DL según diversas características de la población. 
Tabla 3. Desempeño laboral según sexo, hospital donde labora, área específica y tipo de contrato del tecnólogo médico en el área de radiología en HNERM-HNGAI-2016

Table 3. Employee performance of radiology technicians at HNERM-HNGAI, 2016, by sex, employer, specific department, and type of contract

\begin{tabular}{|c|c|c|c|c|}
\hline Características & Regular & Bueno & Muy bueno & Total general \\
\hline \multicolumn{5}{|l|}{ Género } \\
\hline$\%$ Femenino & $3.12 \%$ & $12.50 \%$ & $26.56 \%$ & $42.19 \%$ \\
\hline \% Masculino & $4.69 \%$ & $15.63 \%$ & $37.50 \%$ & $57.81 \%$ \\
\hline$\%$ Total general & $7.81 \%$ & $28.13 \%$ & $64.06 \%$ & $100 \%$ \\
\hline \multicolumn{5}{|l|}{ Hospital donde labora } \\
\hline$\%$ HNERM & $4.69 \%$ & $9.38 \%$ & $43.75 \%$ & $57.81 \%$ \\
\hline$\% \mathrm{HNGAI}$ & $3.12 \%$ & $18.75 \%$ & $20.31 \%$ & $42.19 \%$ \\
\hline$\%$ Total & $7.81 \%$ & $28.13 \%$ & $64.06 \%$ & $100 \%$ \\
\hline \multicolumn{5}{|l|}{ Área específica } \\
\hline$\%$ RX - INT & $6.25 \%$ & $18.75 \%$ & $42.19 \%$ & $67.19 \%$ \\
\hline$\%$ TOM - RM & $1.56 \%$ & $9.38 \%$ & $21.87 \%$ & $32.81 \%$ \\
\hline$\%$ Total & $7.81 \%$ & $28.13 \%$ & $64.06 \%$ & $100 \%$ \\
\hline \multicolumn{5}{|l|}{ Tipo de contrato } \\
\hline \% Nombrado & $1.56 \%$ & $4.69 \%$ & $17.19 \%$ & $23.44 \%$ \\
\hline \% C. Indeterminado & $3.12 \%$ & $17.19 \%$ & $37.50 \%$ & $57.81 \%$ \\
\hline \% C. Administrativo de Servicios & $3.12 \%$ & $6.25 \%$ & $9.37 \%$ & $18.75 \%$ \\
\hline \% Total & $7.81 \%$ & $28.13 \%$ & $64.06 \%$ & $100 \%$ \\
\hline
\end{tabular}

Fuente: elaboración propia.

Finalmente, en el análisis bivariado se encontró que sí existe una asociación entre el puntaje obtenido de los diversos FM y el DL, se utilizó ambas variables en su forma numérica y mediante la prueba de correlación de Pearson se encontró los coeficientes de correlación de cada FM (Ver tabla 4). 
Tabla 4. Coeficiente de correlación de Pearson al asociar los factores motivacionales con el desempeño laboral en el tecnólogo médico en el área de radiología en HNERM-HNGAl-2016

Table 4. Pearson correlation coefficient that associates the motivating factors with the employee performance of radiology technicians at HNERM-HNGAI, 2016

\begin{tabular}{|c|c|c|}
\hline Factores motivacionales & $\begin{array}{c}\text { Coeficiente de correlación }(r) \text { con el } \\
\text { DL }\end{array}$ & $p$ \\
\hline Crecimiento & 0.0286 & 0.8225 \\
\hline Relaciones con los compañeros & 0.0684 & 0.591 \\
\hline Seguridad laboral & 0.2511 & +0.04 \\
\hline Logro & -0.0665 & 0.601 \\
\hline Condiciones de trabajo & 0.1502 & 0.236 \\
\hline Políticas y administración & -0.0609 & 0.632 \\
\hline Responsabilidad & 0.147 & 0.246 \\
\hline Vida personal & -0.1286 & 0.311 \\
\hline Trabajo en sí mismo & 0.0483 & 0.704 \\
\hline Sueldo & 0.067 & 0.5987 \\
\hline Ascenso & -0.0939 & 0.46 \\
\hline Reconocimiento & -0.1428 & 0.26 \\
\hline Relaciones con jefe & -0.2508 & +0.04 \\
\hline Supervisión & -0.0172 & 0.89 \\
\hline Estatus & 0.0306 & 0.816 \\
\hline
\end{tabular}

Se encontró una correlación estadísticamente significativa solo en los FM de seguridad laboral y relaciones con el jefe. Se evidenció que a mayor priorización del FM de seguridad laboral se presentaba un mayor nivel de DL (correlación positiva débil r: 0.2511, p: 0.04) y que contrariamente, mientras más se priorizaba el factor de calidad de la relación con el jefe, se presentaba un menor nivel de desempeño laboral (correlación negativa débil r: -0.2508, p: 0.04).

Asimismo, no se encontró asociación entre tener un buen o muy buen DL y el pertenecer a un hospital específico, tener un tipo de contrato específico o trabajar en un área específica (Test de Chi Cuadrado: $p=0.33 ; p: 1.00 ; p: 0.44$ respectivamente y un $\mathrm{IC}=95 \%$ ).

\section{DISCUSIÓN}

Es importante determinar los FM asociados al DL; la relación entre ambas variables determina, en mayor o menor grado, la disposición hacia el trabajo desempeñado en el área de salud. Una de las limitaciones que se presentó fue el escaso número de investigaciones realizados con TM y por ello 
en esta discusión, se realiza con grupos ocupacionales de salud y en los servicios asistenciales de salud.

Respecto a los factores motivacionales, Bobadilla Asenjo (2016) realizó su investigación con una población de enfermeras en menor número de muestra comparada a la propia y aplicó un cuestionario enfocado a los FM, con el cual encontró que los factores más importantes fueron crecimiento y relaciones con los compañeros, en similitud con los resultados propios. Por otro lado, dieron menos importancia a la responsabilidad, siendo un hallazgo de aspecto negativo, porque la responsabilidad potencializa los FM en la organización, a diferencia de los resultados propios que muestran la responsabilidad considerado como uno de los más importantes, siendo estos debido al grupo ocupacional en estudio.

En comparación con Espino Ruiz (2014), que realizó su investigación con una población mayor a la del presente trabajo y aplicó un test diferente pero enfocado a los FM, en tres grupos de trabajo: profesionales, técnicos y auxiliares. El sueldo, las relaciones con los compañeros y con el jefe, fueron considerados más importantes por los técnicos, siendo estos resultados semejantes a los resultados propios; la condición de trabajo y ascenso fue contemplado más importante por los profesionales, siendo estos resultados también similares a los resultados propios.

Arboleda Posada y Cardona Jiménez (2018) realizaron su estudio con una población en mayor número de muestra comparada a la de esta investigación, aplicando un cuestionario distinto, pero enfocado a los FM, encontrando parecido con esta investigación respecto al grupo etario (30 a 49 años) y que el ambiente de trabajo es el FM más importante, seguido del salario y el cargo, a diferencia que los resultados propios que fueron crecimiento profesional, relaciones con los compañeros y logro; resultados diferentes debido a la infraestructura donde fue realizado el estudio, teniendo en cuenta que en el país de Colombia, en el lugar donde fue realizado el estudio, la necesidad de un ambiente de trabajo es de mayor consideración.

Yongbeom (2018) realizó su estudio con una población mayor a la de esta investigación y los resultados estuvieron basados en la prueba de la teoría de la motivación de F. Herzberg, encontrando que los FM más importantes en los gestores públicos fueron: crecimiento (oportunidades para avance), capacitación y desarrollo profesional, mayor responsabilidad, orgullo de trabajar para la organización, pensión y plan de jubilación, los beneficios y el salario; otros factores: seguridad laboral, similar a la asociación con el DL en el presente estudio.

En el FM crecimiento los gerentes no muestran efectos motivadores significativos, ya que ellos ocupan cargos de gerentes y algunos han alcanzado el puesto más alto de su carrera; en contrastación con los resultados propios, el estatus fue considerado con menos importancia, debido a que los HNERM y HNGAl, en su estructura organizacional de recursos humanos, promueve al empleado, el compromiso de ser parte de una organización de éxito, cubriendo sus aspiraciones, brindándoles la oportunidad de poner en práctica sus conocimientos y capacidades, sintiendo prestigio al pertenecer a EsSalud, esta actitud ha ayudado a fortalecer el cumplimiento de las metas trazadas en las diferentes áreas en donde realiza su función.

En sus investigaciones Purohit y Bandyopadhyay (2014) realizaron su estudio con una población mayor a la del presente estudio y aplicaron un instrumento basado en la teoría motivacional de 
Herzberg y similar a este, encontrando que la «seguridad laboral» fue el factor más importante, dato en similitud con los resultados propios de asociación, seguido por un «trabajo interesante», "respeto y conocimiento» $(p<0.01)$. En la presente investigación el factor motivacional con mayor puntaje de priorización fue el crecimiento y las relaciones con los compañeros, resultados diferentes, debido al escenario físico laboral donde fueron estudiados.

Kiruja y Mukuru (2013) realizaron su estudio con una población mayor a la de este estudio, los resultados concuerdan con los hallazgos en los FM de F. Herzberg, encontrando similitud con la presente investigación respecto al grupo etario, entre 41 a 50 años, estando frente a una población adulta; sin embargo, encontraron que no hay satisfacción en el salario y el entorno laboral, esto puede entenderse como una particularidad en el país de Kenia la cual tiene factores económicos, como déficit presupuestario y un índice de crecimiento ralentizado, mientras que Perú tiene estabilidad financiera, crecimiento público y privado que brinda seguridad económica. A pesar que el grupo etario es similar, la diferencia de resultados en los FM es debido al escenario geográfico de estudio.

En cuanto al FM «responsabilidad», en los resultados propios se evidencia que el profesional TM en el área de radiología, presenta este FM como uno de los más importantes, siendo un valor inherente de cada persona, la cual induce a un compromiso e involucramiento que puede tener el empleado, asumir y ejecutar el cumplimiento de alguna tarea o función, y que coadyuvará a favor de los objetivos de la organización.

Siendo el factor responsabilidad un pilar para poder empezar a potencializar los FM, ya que lo lleva a la búsqueda constante de la superación en el ámbito tecnológico, donde la velocidad de cambios es vertiginosa, y los adelantos en el equipamiento de imagenología es cada año cambiante, hay mejores equipos médicos computarizados de mayor resolución y el profesional TM debe de estar dispuesto a desaprender y aprender nuevamente para aplicarlo correctamente, motivándose a desarrollar y perfeccionar la habilidad de la adquisición de las mejores imágenes, para armonizar con el equipo médico hacia un diagnostico eficaz y eficiente, en favor del paciente.

Referente al DL, la presente investigación considera dos dimensiones y ocho indicadores, basados en la evaluación práctica del escenario físico donde es realizado la actividad profesional, las actitudes personales y el trabajo en equipo donde se labora, en su contenido están: rendimiento laboral (con indicadores: cumplimiento de metas asignadas y calidad de trabajo efectuado) e idoneidad en el trabajo (con indicadores: competencia para el puesto, servicio y atención al paciente, compromiso y responsabilidad, nivel de integración y trabajo en equipo, criterio de urgencia e importancia, y habilidad de gestión bajo presión).

A diferencia de la presente investigación, Cancha Mendez y Yauri Castro (2019) considera el DL con cuatro dimensiones: capacidad técnica (con indicadores: cumplimiento de protocolos, aplicación del proceso de atención y trabajo en equipo), capacitación y educación continua (con indicadores: participación como asistente o ponente, y realización de investigaciones), productividad (con indicadores: trabajo con eficiencia, eficacia y efectividad) y aptitudes (con indicadores: identificación de problemas, adaptaciones a los cambios, necesidades de la institución y generación de acciones de iniciativas laborales). Sin embargo, existen similitudes con los resultados propios al concluir que los 
factores motivacionales tienen asociación significativa con el desempeño laboral de los profesionales de enfermería.

Campos Huarez (2018) considera el DL con tres dimensiones: logro de metas (con indicadores: eficacia, eficiencia, efectividad), competencias (con indicadores: habilidades y destrezas, compromisos con objetivos y metas institucionales, responsabilidad e iniciativa), y rasgos de la personalidad (con indicadores: sociabilidad y autocontrol). Resultados: eficiente $20 \%$, regular $65 \%$ y deficiente $15 \%$.

Mientras tanto, Fuentes Morillo (2018) valúa el DL con dos dimensiones: adquisición de capacidades (con indicadores: perfil del cargo, conocimiento del puesto, pericia y experiencia), y adquisición de valores éticos (con indicadores: compromiso, responsabilidad, pro actividad y colaboración). Resultados: adquisición de capacidades $66 \%$ y adquisición de valores éticos $68 \%$.

Bobadilla Asenjo (2016) evalúa el DL con una dimensión: Competencias (con los indicadores: actitudes, conocimiento, y habilidades y destrezas); mayor importancia a las actitudes (83.3\%), luego conocimiento (16.7\%) y por último las habilidades y destrezas (4.2\%).

En los resultados de la presente investigación hay asociación de los FM y el DL, en los FM «Relación con el jefe» y «Seguridad laboral», y en los demás FM no se encuentra una diferencia significativa de asociación. En el FM «relaciones con el jefe», la asociación es de manera inversamente proporcional; es decir, mientras existan menos calidad de «relaciones con el jefe» y el TM en el área de radiología, mayor será el DL, y viceversa. Se considera que la efectividad de una buena relación entre el TM y la organización dependerá mucho de la relación TM y jefe inmediato superior; siendo el jefe inmediato superior o coordinador, el que tenga la habilidad para influenciar y motivar a que los empleados aporten a favor de las metas y de los objetivos de la organización.

Teniendo en los resultados propios un efecto negativo de DL frente a que los TM en el área de radiología que más priorizan la calidad de «relaciones con el jefe», esto debido a que, durante la investigación, en EsSalud hubo cambios de jefatura y dirección, la cual no brindaron una base primordial para el cumplimiento de sus objetivos, agregando que los TM no les interesó mucho obtener resultados a partir de la jefatura y trabajaron mejor independientemente de la jefatura.

El FM «seguridad laboral», asociación directamente proporcional; es decir, a mayor percepción de seguridad laboral del TM en el área de radiología, mayor será su DL, y viceversa; esto debido a que la organización en EsSalud les brinda estabilidad emocional, siendo institución de prestigio que cubre casi al $100 \%$ de las expectativas, por tener equipos de avanzada tecnología, mostrando que estos FM se encuentran en complacencia y no resalta importancia a los otros FM.

A su vez, los resultados obtenidos en la seguridad laboral, expresa que el TM en el área de radiología, muestra preocupación por mantenerse estable en el trabajo, ya que EsSalud, durante la investigación, brinda diferentes condiciones de trabajo, siendo la modalidad CAS, la más endeble en estabilidad de trabajo; esta preocupación resaltaría este FM, mostrando importancia pero con efecto de intranquilidad y desconfianza en conservar su trabajo en la organización, buscando identificarse institucionalmente y demostrar eficiencia y eficacia en su empresa. 
Se brinda estos resultados para la gestión de recursos humanos y promover las actividades de superación en el profesional TM, creando planes de mejora para el fortalecimiento de las relaciones interpersonales y la organización.

\section{CONCLUSIONES}

Los FM de mayor significancia e importancia Para los TM del el área de radiología del HNERM y el HNGAI son el crecimiento profesional, las relaciones con los compañeros, el logro y la seguridad laboral; factores que reflejan la existencia real de posibilidades de promoción como un estímulo positivo y atractivo, buena comunicación interna que mejora la cohesión y relaciones entre sus miembros, buena autoestima personal con el puesto de trabajo actual, y estabilidad laboral que generan un buen rendimiento.

El DL de los TM del área de radiología de los hospitales HNERM y HNGAI (de acuerdo con la evaluación de los jefes inmediatos) es bueno (28.13\%) y muy bueno (64.06\%), datos que indican un buen rendimiento en el centro de labores, y que refuerzan los resultados obtenidos acerca de los FM.

Existe asociación significativa entre los FM «seguridad laboral», «relaciones con el jefe» y el DL en los TM del área de radiología. Donde se evidenció que, a mayor priorización del FM de las relaciones con el jefe, menor es el nivel de DL; y que contrariamente, a mayor priorización del FM seguridad laboral, mayor es el nivel de DL. En consecuencia, se puede inferir que es gracias a esta asociación significativa que el DL sea catalogado preponderantemente como bueno y muy bueno, y que el rendimiento laboral sea óptimo. Por otro lado, no se puede dejar de mencionar que sería un reto importante realizar futuras investigaciones para analizar qué es lo que realmente pasa con los 13 FM restantes que no presentaron asociación significativa.

\section{AGRADECIMIENTOS}

El equipo investigador quiere agradecer la colaboración prestada por los directivos; el Comité de Capacitación, Investigación y Docencia del Hospital Nacional Edgardo Rebagliati Martins y el Hospital Nacional Guillermo Almenara Irigoyen por brindar facilidades para la ejecución de la investigación.

\section{REFERENCIAS}

Alvarado Arévalo, E. F. (2017). Motivación laboral como factor de disminución de rotación de personal en las organizaciones (Trabajo de grado de especialización). Universidad Militar Nueva Granada, Bogotá, Colombia.

Anderson, T. J.; Mendes de Barros, A. M.; da Silva Copelli, F. H.; Coelho de Melo, J. A. (2016). Occupational risks of radiology technicians in care for patients with multiple trauma. O Mundo da Saúde, v. 40, n. 1, 106-113. https://doi.org/10.15343/0104-7809.20164001106113 
Arana Mayorca, W. H. (2004). Diseño y Validación de un modelo para la identificación y medición de los Factores Motivacionales de los trabajadores según la Teoría de Frederick Herzberg (Tesis de maestría) Universidad Nacional Mayor de San Marcos, Lima, Perú.

Arboleda Posada, G. I.; Cardona Jiménez, J. L. (2018). Percepción de la satisfacción con la labor desempeñada y factores de motivación del personal de las instituciones prestadoras de servicios de salud (IPS) del Valle de Aburrá, 2011. Gerencia y Políticas de Salud, v. 17, n. 35. 222-234. http://dx.doi.org/10.11144/javeriana.rgps17-35.psld

Arias Gonzales, G. (2019). La motivación laboral y el desempeño laboral de los trabajadores del centro de salud San Atancio de Pedregal, Lima, 2018 (Trabajo de grado). URL

Billyard, A. P.; Donohue, J. J. (2016). A practical organizational efficiency measure. Defence and Peace Economics, v. 27, n. 6, 794-819. https://doi.org/10.1080/10242694.2014.987902

Bobadilla Asenjo, E. G. (2016) Factores motivacionales y desempeño laboral del profesional de Enfermería en el Servicio de Emergencia de un Hospital de ESSALUD Chiclayo. Abril-octubre, 2016 (Tesis de maestría). Universidad Cesar Vallejo, Lima, Perú.

Cancha Mendez, J. E.; Yauri Castro, T. S. (2019). Factores asociados al desempeño laboral de los profesionales de enfermería-hospital Víctor Ramos Guardia-Huaraz-2018 (Trabajo de grado). $\underline{U R L}$

Campos Huarez, M. A. (2018). Factores motivacionales en el desempeño laboral del profesional de enfermería del servicio de emergencia del Hospital Vitarte Lima - 2017 (Tesis de maestría). URL

Chiavenato, I. (2009). Comportamiento organizacional La dinámica del éxito en las organizaciones. McGraw - Hill.

Espino Ruiz, A. Y. (2014). Los factores motivacionales que influyen en el desempeño laboral del personal administrativo del Hospital Regional de Cajamarca: una propuesta de programa de motivación - 2014 (Trabajo de grado). URL

Franco-López, J. A.; Bedoya-Zapata, J. (2018). Análisis de modelos del talento humano aplicados en organizaciones pymes. Revista CEA, v. 4, n. 7, 85-101. https://doi.org/10.22430/24223182.761

Fuentes Morillo, J. M. (2018) La motivación y el desempeño laboral de los empleados de la Oficina Registral de Propiedad Inmueble de la Zona Registral $N^{\circ} I X$ - Sede Lima de la Superintendencia Nacional de los Registros Públicos SUNARP, 2017 (Tesis de maestría). URL

García Santillán, A.; Uscanga Guevara, M. T. (2008). Desarrollo y comportamiento de la motivación en el trabajo. $\underline{\text { URL }}$

Herzberg, F. (2003). Una vez más, icómo motiva a sus empleados? Harvard Business Review, Edición América Latina, v. 81, n. 1, 67-76. 
Jiang, W.; Chai, H.; Li, Y.; Feng, T. (2019). How workplace incivility influences job performance: the role of image outcome expectations. Asia Pacific Journal of Human Resources, v. 57, n. 4, 445469. https://doi.org/10.1111/1744-7941.12197

Kiruja, E. K.; Mukuru, E. (2013). Effect of Motivation on Employee Performance in Public Middle Level Technical Training Institutions in Kenya. International Journal of Advances in Management and Economics, v. 2, n. 4, 73-82. URL

Linares Marín, J. S. (2017). Motivación laboral y desempeño laboral en el centro de salud la Huayrona 2017 (Tesis de maestría). URL

López Más, J. (2005). Motivación laboral y gestión de recursos humanos en la teoría de Frederick Herzberg. Gestión En El Tercer Milenio, v. 8, n. 15, 25-36.

Lusthaus, C.; Adrien, M. H.; Anderson, G.; Carden, F.; Montalván, G. P. (2002). Evaluación organizacional: marco para mejorar el desempeño. Banco Interamericano de Desarrollo.

Marín Samanez, H. S.; Placencia Medina, M. D. (2017). Motivación y satisfacción laboral del personal de una organización de salud del sector privado. Horizonte Médico (Lima), v. 17, n. 4, 42-52. https://doi.org/10.24265/horizmed.2017.v17n4.08

Martin, H.; Sorhaindo, C. (2019). A comparison of intrinsic and extrinsic motivational factors as predictors of civil engineering students' academic success. International Journal of Engineering Education, v. 35, n. 2, 458-472. URL

Nojedeh, S. H. (2015). Identifying and prioritizing the indicators of talent management in recruiting radiology technicians. International Journal of Organizational Leadership, v. 4, n. 3, 213-224. https://doi.org/10.33844/ijol.2015.60215

Organización Mundial de la Salud. (2010). Entornos Laborales Saludables: Fundamentos y Modelo de la OMS, Contextualización, Prácticas y Literatura de Apoyo. $\underline{\text { URL }}$

Palaci Descals, F. J. (2005). Psicología de la Organización. Pearson Prentice Hall.

Paredes Cabanillas, E. I. (2017). Motivación y desempeño laboral en personal del departamento de patología clínica del H.N.H.U. El Agustino 2016 (Tesis de maestría). Universidad Cesar Vallejo, Lima, Perú

Peiró, J. M.; Meliá, J. L. (2003). Formal and informal interpersonal power in organisations: Testing a bifactorial model of power in role-sets. Applied Psychology, v. 52, n. 1, 14-35. https://doi.org/10.1111/1464-0597.00121

Purohit, B.; Bandyopadhyay, T. (2014). Beyond Job Security and Money: Driving Factors of Motivation for Government Doctors in India. Human Resources for Health, v. 12, 1-13. https://doi.org/10.1186/1478-4491-12-12 
Ríos Valdera, J. D. (2017). Satisfacción y desempeño laboral del profesional de salud en la sede de la gerencia regional de salud Lambayeque-2017 (Tesis de maestría). Universidad Cesar Vallejo, Lima, Perú

Robbins, S. P. (2004). Comportamiento Organizacional. Pearson Educación, México 2004.

Román Meza, A. H. (2016). Satisfacción del tecnólogo médico de radiología en la Clínica Internacional - Sede San Borja en el año 2015 (Tesis de maestría). Universidad Nacional Mayor De San Marcos, Lima, Perú.

Salcedo Ramos, I. J. (2020) Factores institucionales y el desempeño de tecnólogos médicos de laboratorio clínico del Instituto Nacional de Enfermedades Neoplásicas, Lima 2019 (Tesis de maestría). Universidad Cesar Vallejo, Lima, Perú.

Seguro Social de Salud EsSalud. (2004). Resolución de presidencia ejecutiva oo 1046 -pe-essalud2004. URL

Seguro Social de Salud EsSalud. (2016). Análisis ejecutivo a nivel nacional de las prestaciones de salud 2016. URL

Seguro Social de Salud EsSalud. (2020). Nuestra Institución. URL

Silva Rodríguez de San Miguel, J. A. (2018). La gestión y el desarrollo organizacional: marco para mejorar el desempeño del capital humano. $\underline{\text { URL }}$

Uribe-Gómez, J. A. (2018). Aproximación sistémica al dimensionamiento de personal en instituciones prestadoras de servicios de salud. Revista CEA, v. 4, n. 8, 51-65. https://doi.org/10.22430/24223182.1047

Vänje, A. (2015). Sick Leave-A Signal of Unequal Work Organizations? Gender perspectives on work environment and work organizations in the health care sector: a knowledge review. Nordic Journal of Working Life Studies, v. 5, n. 4, 85-104. https://doi.org/10.19154/njwls.v5i4.4845

Vázquez-Moctezuma, S. E. (2014) La motivación de los empleados en bibliotecas a través de la teoría de las expectativas. Revista Infoacceso, v. 2, n. 1, 3-14.

Yongbeom, H. (2018). Testing Herzberg's Two-Factor Theory of Motivation in the Public Sector: Is it Applicable to Public Managers? Public Organization Review, v. 18, n. 3, 329-343. https://doi.org/10.1007/s11115-017-0379-1

Yunus, M.; Lis, E. Y.; Adam, M.; Sofyan, H. (2020). Does Motivation Mediate the Effects of Employee Staff Empowerment, Talent, Working Environment, and Career Development on Staff Performance? Quality-Access to Success, v. 21, n. 175. URL 